\title{
Reliability Analysis for FDDI Dual Homing Networks
}

\author{
Gam D. Nguyen \\ Naval Research Laboratory, Washington DC 20375
}

\begin{abstract}
Closed-form reliability functions for the well-known dual homing configuration for the Fiber Distributed Data Interface (FDDI) are derived by the familiar combinatorial method. The new reliability functions are then compared to that of the dual ring configuration via numerical examples, which again show that in most cases the dual homing network is more reliable than the dual ring network; however, the model analysis also shows that dual homing is recommended only if certain network parameter constraints are met.
\end{abstract}

\section{Introduction}

Fiber Distributed Data Interface (FDDI) is a high-speed, fiber-optic token network consisting of two counter-rotating rings [1]. In addition to the fault tolerant level provided by the dual rings, the reliability of an FDDI network is enhanced by the use of station bypass switches or concentrators (CONs) $[1,2,3]$. A station equipped by a bypass switch is switched out of the ring when the station experiences a power failure. A CON facilitates the connection of stations to the ring and also switches out of the ring any faulty station connected to it. The use of reliable CONs to interconnect stations is the heart of the dual homing configuration.

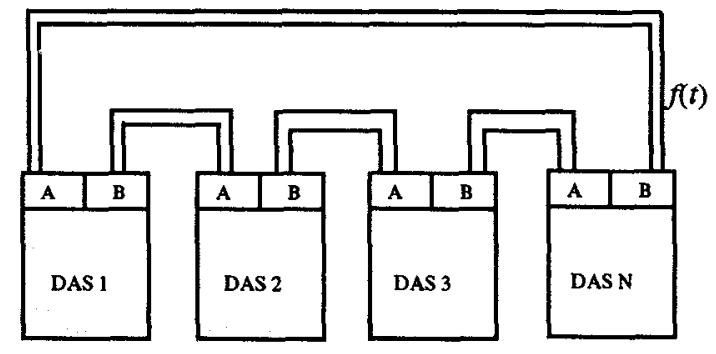

Fig. 1 - Dual ring network
The simplest way to form an FDDI network of $N$ dual attach stations (DASs) is to interconnect the stations as shown in Fig. 1. To use the reliability provided by two counter-rotating rings, DASs must be used in the FDDI network. The DASs can be any type of dual attach nodes such as gateways, CONs, or servers. This simple configuration (Fig. 1) is called the dual ring network. The reliability function $r(t)$ of the dual ring is given in Ref. 2. Suppose that optical bypass switches in the DASs are working perfectly, then

$$
r(t)=N f(t)^{2 N-2}-(N-1) f(t)^{2 N},(1)
$$

where $N$ is the number of DASs and $f(t)$ is the reliability function of one of the $2 N$ links. A dual homing analogy of $\mathrm{Eq}$. (1) is derived in the next section. Equation (1) is valid under the assumption that the dual ring is reliable as long as the ring is not segmented. Optical bypass switches in DASs switch any station that has no power out of the ring; therefore, the reliability of the ring is not affected unless the number of activated optical bypass switches is excessive. However, the loss caused by activated bypass switches affects both the dual ring and the dual homed network almost equally. This paper will not take the DAS faults into consideration. Keep in mind that these results can be extended straightforwardly to incorporate the DAS faults into the model as is done in Ref. 2.

$$
\hat{f}(t)
$$

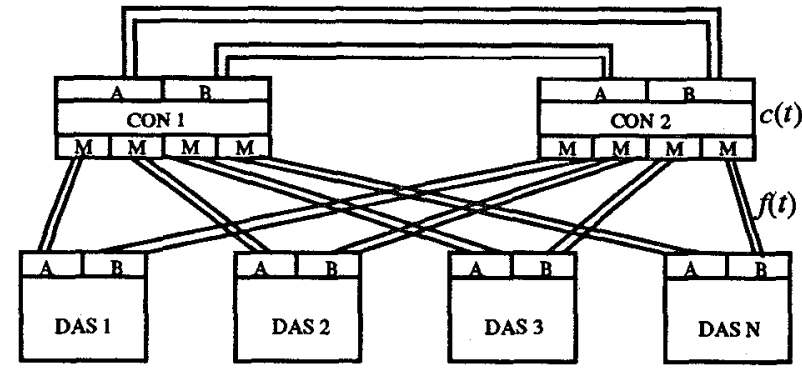

Fig. 2- Dual homing network 
An alternative for the dual ring network is the dual homing network, which is shown in Fig. 2. As expected, the reliability of the dual homing network is improved in many cases by using two additional reliable CONs and $4(N+1)$ fiber links instead of using only the $2 N$ fiber links that were used in the dual ring network. The quantification of this observation is given in Sections 2 and 3. In addition to providing further reliability, the dual homing network is highly structured and hence facilitates network management as well as network expansion/reduction; stations may be added to or removed from the network without altering its structure.

The FDDI dual homing architecture has been proposed, studied, and implemented for several years. The comparison between dual homing and other types of configurations is reviewed in Ref. 3. The availability of the path between user and backbone is discussed in Ref. 4 for multiple level FDDI dual homing. The goal of this paper is to derive reliability results of the dual homing network shown in Fig. 2 (as well as its extensions shown in Fig. 3), where each CON has an arbitrary reliability function $c(t)$, $N$ is the number of DASs, each of the four links connecting the two CONs has a general reliability function $\hat{f}(t)$, and each of the $4 N$ links connecting DASs to the two CONs has an arbitrary reliability function $f(t)$. It is assumed that all components fail independently and that each CON is capable of handling $N$ DASs. It is important to note that the link fault can be any fault that causes the ring to wrap or to be segmented and can include many types such as fiber cuts, severe $\mathrm{dB}$ loss, or faulty station transceivers. This simple dual homing model is fundamental for the following reasons:

- The model is the dual homing counterpart of the dual ring shown in Fig. 1. Most FDDI backbone rings can be transformed into either one of these two configurations since FDDI standards require that the FDDI backbone be connected by dual attach nodes.

- Many other FDDI configurations involving dual homing can be analyzed by using this dual homing model as the basic building block (Sections 2 and 3); with the use of only one pair of CONs as shown in Fig. 2, the insight can be gained more easily from this simple dual homed network model.

- It is desirable to have a closed form and yet simple formula, which is a dual homing counterpart of Eq. (1) and can be computed in a straightforward manner (Theorem 1).

- The model analysis shows that dual homing is beneficial only if certain network parameter constraints are met (Theorem 2).

\section{Reliability Results for Dual Homing Network of Figure 2}

This section is devoted to the derivation of the reliability function for the dual homing network posed in Section 1. Then a numerical example is given to apply the derived formula, followed by a theorem about network parameter constraints for a proper dual homing implementation. A more general model is analyzed in the next section.

Let $f(t), \hat{f}(t), c(t)$, and $R(t)$ be reliability functions of the link connecting DASs to the CONs, of the link connecting two CONs, of the CON, and of the dual homing network respectively (Fig. 2). Furthermore, let $N$ be the number of DASs. The network is said to be operational (i.e., reliable) if there is a communication path among all DASs (i.e., the network is not segmented).

\section{Theorem 1}

$$
\begin{aligned}
& R(t)=\left(\left(2 f(t)^{2}-f(t)^{4}{ }^{N}\left[2 \hat{f}(t)^{2}-\hat{f}(t)^{4}\right]\right.\right. \\
& \left.\quad+f(t)^{2 N}\left[1-\hat{f}(t)^{2}\right]^{2}\right) c(t)^{2}+f(t)^{2 N} 2 c(t)[1-c(t)] .
\end{aligned}
$$

Proof: Let $X$ be the random variable representing the operational time of the dual homing network shown in Fig. 2. For each $t>0$, consider two mutually exclusive and exhaustive events $A(t)$ and $B(t)$ for the CONs: $A(t)$ is the event that two CONs are still operational at time $t$ and $B(t)$ is the event that only one CON is still operational at time $t$. Then

$$
\begin{aligned}
& \operatorname{Pr}\{A(t)\}=c(t)^{2} \text { and } \\
& \quad \operatorname{Pr}\{B(t)\}=2 c(t)[1-c(t)] .
\end{aligned}
$$

Let $A_{1}(t)$ be the event that the root ring (i.e., the ring connecting the two CONs) is not segmented before $t$. Then from Eq. (1) with $N=2$

$$
\operatorname{Pr}\left\{A_{1}(t) \mid A(t)\right\}=2 \hat{f}(t)^{2}-\hat{f}(t)^{4} .
$$

Let $A_{2}(t)$ be the event that the root ring is segmented before $t$. Then from Eq. (3)

$$
\begin{aligned}
\operatorname{Pr}\left\{A_{2}(t) \mid A(t)\right\} & =1-\operatorname{Pr}\left\{A_{1}(t) \mid A(t)\right\} \\
& =\left[1-\hat{f}(t)^{2}\right]^{2} .
\end{aligned}
$$

Then $\operatorname{Pr}\left\{X>t \mid A(t), A_{1}(t)\right\}$ is computed as follows: With the presence of the two operational CONs, effec- 
tively the fiber links always fail in pairs with a new reliability function $f(t)^{2}$ for each pair. Each DAS is disconnected from the dual homed network when both pairs (each with reliability function $f(t)^{2}$ ) fail; that is, the reliability associated with each DAS is

$$
1-\left[1-f(t)^{2}\right]^{2}=2 f(t)^{2}-f(t)^{4} .
$$

Since there are $N$ such DASs,

$$
\operatorname{Pr}\left\{X>t \mid A(t), A_{1}(t)\right\}=\left[2 f(t)^{2}-f(t)^{4}\right]^{N} .
$$

Eq. (5) is used several times in the computation in the next section.

For the case where the root ring is segmented, which results in two identical rings of single attach stations (SASs), $\operatorname{Pr}\left\{X>t \mid A(t), A_{2}(t)\right\}$ is computed as follows: Note that the second ring is no longer considered reliable because if it is reliable, then all of the links at port $B$ would have to fail simultaneously; this is impossible as $f(t)$ is associated with a continuous random variable by assumption. Port B links are always active unless there are faults associated with them. Therefore,

$$
\operatorname{Pr}\left\{X>t \mid A(t), A_{2}(t)\right\}=f(t)^{2 N} .
$$

From Eqs. (3) to (6) and the fact that

$\operatorname{Pr}\{X>t \mid A(t)\}$

$$
\begin{aligned}
& =\operatorname{Pr}\left\{X>t \mid A(t), A_{1}(t)\right\} \operatorname{Pr}\left\{A_{1}(t) \mid A(t)\right\} \\
& +\operatorname{Pr}\left\{X>t \mid A(t), A_{2}(t)\right\} \operatorname{Pr}\left\{A_{2}(t) \mid A(t)\right\},
\end{aligned}
$$

$\operatorname{Pr}\{X>t \mid A(t)\}$

$$
\begin{aligned}
& =\left[2 f(t)^{2}-f(t)^{4}\right]^{N}\left[2 \hat{f}(t)^{2}-\hat{f}(t){ }^{4}\right] \\
& +f(t)^{2 N}\left[1-\hat{f}(t)^{2}\right]^{2} .
\end{aligned}
$$

Note that

$\operatorname{Pr}\{X>t \mid B(t)\}=f(t)^{2 N}$.

Then from Eqs. (2), (7), (8) and the fact that

$$
\begin{aligned}
R(t)=\operatorname{Pr}\{X>t\}=\operatorname{Pr}\{X>t \mid A(t)\} \operatorname{Pr}\{A(t)\} \\
+\operatorname{Pr}\{X>t \mid B(t)\} \operatorname{Pr}\{B(t)\},
\end{aligned}
$$

$$
\begin{aligned}
R(t) & =\left(\left(2 f(t)^{2}-f(t)^{4}{ }^{N}\left[2 \hat{f}(t)^{2}-\hat{f}(t)^{4}\right]\right.\right. \\
& \left.+f(t)^{2 N}\left[1-\hat{f}(t)^{2}\right]^{2}\right) c(t)^{2}+f(t)^{2 N} 2 c(t)[1-c(t)] .
\end{aligned}
$$

\section{Remarks}

- Suppose that two perfect CONs with $c(t)=1$ are used and that the root ring is not segmented (i.e., $\hat{f}(t)=1$ ). Then Theorem 1 becomes

$$
R(t)=\left[2 f(t)^{2}-f(t)^{4}\right]^{N},(9)
$$

which must be an upper bound for the reliability function $R(t)$ of the dual homing network. This upper bound can be approached as close as desirable by using two very reliable CONs as well as four very reliable (e.g., very short) links.

- The reliability function in Theorem 1 is dependent on $\hat{f}, f$, and $c$; i.e., $R=R(f, \hat{f}, c)$ (here, for ease of writing, the time variable $t$ is suppressed). Then an upper bound for the reliability function $R$, which is tighter (and more complicated) than Eq. (9), is given by

$$
\begin{aligned}
& R \leq \min (R(1, \hat{f}, c), R(f, 1, c), R(f, \hat{f}, 1), \\
&R(f, 1,1), R(1, \hat{f}, 1), R(1,1, c)) .(10)
\end{aligned}
$$

Note that $R(f, 1,1)$ is the same as Eq. (9).

\section{Example 1}

Suppose that $\hat{f}(t)=c(t)=f(t)=e^{-0.001 t}$, where $t$ is the time measured, for instance, in days. This will give the mean time to failure (MTTF) of both the CON and fiber link the value of $1 / 0.001=1000$ days. Then the reliability of the dual ring as well as the dual homing network for various values of $t$ and $N$ (the number of DASs) is given in Table 1 ( $M$ is the number of CON pairs, $M=1$ in this example).

From Table 1, for small network sizes (e.g., $N=5$ ), the dual ring network is slightly more reliable than the dual homing network since it is less likely that the small dual ring will be segmented. For larger values of $N(N>9)$, dual homing consistently becomes more reliable than dual ring. Finally, as expected, the reliability upper bound value for dual homing is the greatest among the three values. The following theorem confirms the superiority of dual homing (Eq. (9)) to dual ring (Eq. (1)) when dual homing is properly implemented (i.e., when $N \geq 4$ and the reliability upper bound (Eq. (9)) is approached). 
Table 1 - Reliability of Dual Homing and Dual Ring Network

\begin{tabular}{|c|c|c|c|}
\hline$M=1$ & $t=5$ & $t=10$ & $t=50$ \\
\hline \multirow[t]{3}{*}{$N=5$} & $0.9995^{1}$ & 0.9980 & 0.9555 \\
\hline & $0.9990^{2}$ & 0.9961 & 0.9200 \\
\hline & $0.9990^{3}$ & 0.9962 & 0.9255 \\
\hline \multirow[t]{3}{*}{$N=10$} & 0.9990 & 0.9961 & 0.9130 \\
\hline & 0.9980 & 0.9925 & 0.8577 \\
\hline & 0.9958 & 0.9841 & 0.7548 \\
\hline \multirow[t]{3}{*}{$N=15$} & 0.9985 & 0.9941 & $\overline{0.8724}$ \\
\hline & 0.9971 & 0.9890 & 0.8062 \\
\hline & 0.9905 & 0.9653 & 0.5751 \\
\hline \multirow[t]{3}{*}{$\bar{N}=20$} & 0.9980 & 0.9922 & $\overline{0.8336}$ \\
\hline & 0.9962 & 0.9857 & 0.7621 \\
\hline & 0.9833 & 0.9411 & 0.4200 \\
\hline \multirow[t]{3}{*}{$N=21$} & 0.9979 & 0.9918 & 0.8261 \\
\hline & 0.9960 & 0.9850 & 0.7531 \\
\hline & 0.9817 & 0.9358 & 0.3929 \\
\hline \multirow[t]{3}{*}{$N=30$} & 0.9970 & 0.9883 & $\overline{0.7612}$ \\
\hline & 0.9945 & 0.9795 & 0.6879 \\
\hline & 0.9642 & 0.8814 & 0.2069 \\
\hline \multirow[t]{3}{*}{$\bar{N}=39$} & 0.9961 & 0.9848 & 0.7013 \\
\hline & 0.9929 & 0.9742 & 0.6309 \\
\hline & 0.9424 & 0.8196 & 0.1033 \\
\hline \multirow[t]{3}{*}{$N=40$} & 0.9960 & 0.9844 & 0.6950 \\
\hline & 0.9928 & 0.9737 & 0.6251 \\
\hline & 0.9398 & 0.8124 & 0.0954 \\
\hline \multirow[t]{3}{*}{$N=48$} & 0.9953 & 0.9814 & 0.6462 \\
\hline & 0.9915 & 0.9692 & 0.5802 \\
\hline & 0.9173 & 0.7542 & 0.0498 \\
\hline \multirow[t]{3}{*}{$N=50$} & 0.9951 & 0.9806 & 0.6345 \\
\hline & 0.9912 & 0.9683 & 0.5697 \\
\hline & 0.9113 & 0.7395 & 0.0422 \\
\hline
\end{tabular}

1 computed from Eq. (9): reliability upper bound for dual homing network

${ }^{2}$ computed from Theorem 1: reliability for dual homing network

${ }^{3}$ computed from Eq. (1): reliability for dual ring network

\section{Theorem 2}

(a) $N f^{2 N-2}-(N-1) f^{2 N}>\left(2 f^{2}-f^{4}\right)^{N}$

for $N=1,2,3$ and all $f \in(0,1)$. That is, a small dual ring network is more reliable than a small dual homing network with the same number of DASs $N=1,2,3$ (see Eq. (9)).

(b) $N f^{2 N-2}-(N-1) f^{2 N}<\left(2 f^{2}-f^{4}\right)^{N}$

for all $N \geq 4$ and all $f \in[\sqrt{2} / 2,1)$. That is, if $c=1$ and $\hat{f}=1$, dual homing is more reliable than dual ring for $N \geq 4$ and $f \geq \sqrt{2} / 2$ (see Eq. (9)).
Proof: (a) is clearly true for ${ }_{3} N=1,2$. For $N=3$, observe that $3\left(1-f^{2}\right)>f^{2}\left(1-f^{2}\right)^{3}$, which can be written after some algebra as

$$
3 f^{4}-2 f^{6}>\left(2 f^{2}-f^{4}\right)^{3}
$$

Therefore (a) is also true when $N=3$.

When $N=4$, (b) becomes $4 f^{6}-3 f^{8}<\left(2 f^{2}-f^{4}\right)^{4}$, which can be written after some algebra as

$$
0<\left(1-f^{2}\right)^{2}\left[\left(-4+11 f^{2}-6 f^{4}\right)+f^{6}\right]
$$

however, $\quad-4+11 f^{2}-6 f^{4} \geq 0$ when $f^{2} \geq 1 / 2$ or $f \geq \sqrt{2} / 2=0.7071 \ldots$. Therefore (b) is true at $N=4$. Note that numerical computation shows that (b) is also true when $N=4$ if $f=0.69183$ and is false if $f=0.69182$. Then (b) is proved for all $N \geq 4$ and all $f \geq \sqrt{2} / 2$ by induction as follows. Suppose that (b) is true at $N$. Then since $\left(1-f^{2}\right)^{2}=1-2 f^{2}+f^{4}>0$, it can be shown after some algebra that

$$
\left[N f^{2 N-2}-(N-1) f^{2 N}\right]\left(2 f^{2}-f^{4}\right)>(N+1) f^{2 N}-N f^{2 N+2} ;
$$

therefore, from induction hypothesis ((b)),

$$
\begin{gathered}
\left(2 f^{2}-f^{4}\right)^{N+1}>(N+1) f^{2 N}-N f^{2 N+2} \\
=(N+1) f^{2(N+1)-2}-N f^{2(N+1)} .
\end{gathered}
$$

Thus (b) is also true at $N+1$; hence the induction proof is completed.

Theorem 2 shows that in most cases, dual homing is more reliable than dual ring; however, to better use the dual homing technique, the following should be met:

- the number of DASs $N$ must be at least four

- the link reliability $f(t)$ is at least $\sqrt{2} / 2=.7071 \ldots$

- the ring comprising two CONs must be reliable.

\section{An Extension}

This section analyzes the dual homing network as shown in Fig. 3, which is a natural extension of the network of Fig. 2. Specifically, let $M$ be the number of CON pairs (one such pair looks like the one shown in Fig. 2). As before, each CON can serve $N$ DASs and has reliability 
function $c(t)$. The ring consisting of $2 M$ CONs is called the root ring.

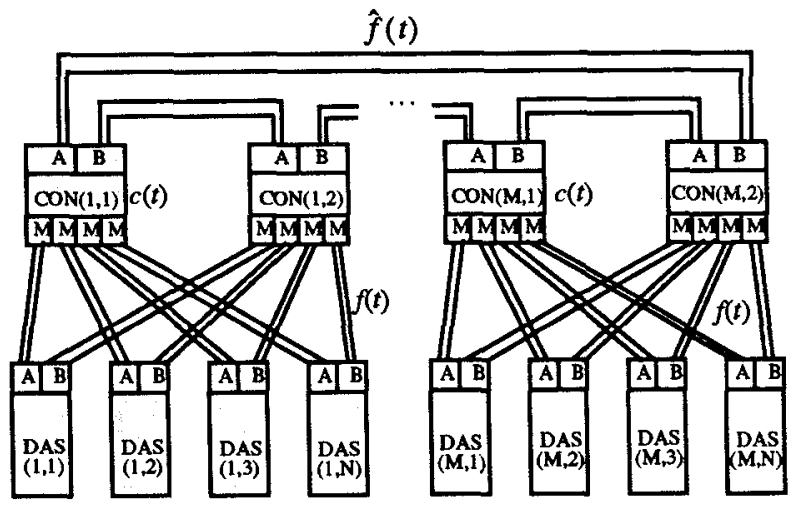

Fig. 3 - Extended dual homing network

Furthermore, each of the (fiber) links used for interconnecting $2 M$ CONs has reliability function $\hat{f}(t)$, and $f(t)$ is the reliability function for each of $4 M N$ links used for connecting DASs to CONs. All components are assumed to fail independently. Other definitions from the previous section such as $X$ and $R(t)$ (Theorem 1) are also carried into this section. Note that the number of DASs now becomes $M N$.

Then the reliability function $R(t)$ of the dual homing network shown in Fig. 3 is given by

$$
\begin{array}{r}
\operatorname{Pr}\{X>t\}=\operatorname{Pr}\{X>t \mid \text { the root ring is not segmented }\} \\
\quad \times \operatorname{Pr}\{\text { the root ring is not segmented }\} \\
+\operatorname{Pr}\{X>t, \text { the root ring is segmented }\} .
\end{array}
$$

Each of the two unknown components in the above summation is computed as follows:

First let $L$ be the number of working CON pairs. Then from the analysis in Section 2 (Eq. (5))

$\operatorname{Pr}\{X>t \mid L=M-m$, the root ring is not segmented $\}$

$$
=\left(\left[2 f(t)^{2}-f(t)^{4}\right]^{N}\right)^{M-m} f(t)^{2 m N} \text { and }
$$

$\operatorname{Pr}\{L=M-m\}=\left(\begin{array}{c}M \\ m\end{array}\right) c(t)^{2(M-m)}(2 c(t)[1-c(t)])^{m}$.

Therefore from the above two equations,

$\operatorname{Pr}\{X>t \mid$ the root ring is not segmented $\}$

$$
=\sum_{m=0}^{M}\left(\left[2 f(t)^{2}-f(t)^{4}\right]^{N}\right)^{M-m} f(t)^{2 m N}
$$

$$
\times\left(\begin{array}{c}
M \\
m
\end{array}\right) c(t)^{2(M-m)}(2 c(t)[1-c(t)])^{m} .
$$

Letting $N=2 M$ in Eq. (1) gives

$\operatorname{Pr}\{$ the root ring is not segmented $\}$

$$
=(1-2 M) \hat{f}(t)^{4 M}+2 M \hat{f}(t)^{4 M-2} .
$$

From the above two equations,

$\operatorname{Pr}\{X>t \mid$ the root ring is not segmented $\}$

$$
\begin{aligned}
\quad \times \operatorname{Pr}\{\text { the root ring is not segmented }\} \\
\sum_{m=0}^{M}\left(\left[2 f(t)^{2}-f(t)^{4}\right]^{N}\right)^{M-m} f(t)^{2 m N} \\
\times\left(\begin{array}{l}
M \\
m
\end{array}\right) c(t)^{2(M-m)}(2 c(t)[1-c(t)])^{m} \\
\times\left[(1-2 M) \hat{f}(t)^{4 M}+2 M \hat{f}(t)^{4 M-2}\right] .(12)
\end{aligned}
$$

Then by the same argument used in the previous section, an upper bound for the reliability function $R(t)$ is

$$
R(t) \leq\left[2 f(t)^{2}-f(t)^{4}\right]^{M N}
$$

Note that the remarks after Theorem 1 as well as Theorem 2 in the previous section remain valid, with slight modification, in this case (Eq. (9)).

The probability $\operatorname{Pr}\{X>t$, the root ring is segmented $\}$ is computed as follows, depending on two cases: $M=2$ and $M \neq 2$.

Case 1: $M=2$. This is the case where there are four CONs. Given the fact that $X>t$ and the root ring is segmented, there are only two types of ring segmentations:

- The ring is segmented evenly resulting in two identical and parallel rings: the first ring consists of $\operatorname{CON}(1,1)$ and $\operatorname{CON}(2,1)$, and the second ring consists of $\operatorname{CON}(1,2)$ and $\operatorname{CON}(2,2)$. Note that the second ring is no longer considered reliable because if it is reliable, then all of the links at port B would have to fail simultaneously, which is an impossible event as $f(t)$ is associated with a continuous random variable. Port $B$ links are always active unless there are faults associated with them.

- The ring is segmented unevenly resulting in two different rings: one ring has only one $\mathrm{CON}$ and the other ring has three CONs. 
First note that for $L=0$,

$\operatorname{Pr}\{X>t, L=0$, the root ring is segmented unevenly $\}$

$=\operatorname{Pr}\{X>t \mid L=0$, the root ring is segmented unevenly $\}$

$\times \operatorname{Pr}\{$ the root ring is segmented unevenly $\mid L=0$ \}

$$
\times \operatorname{Pr}\{L=0\}
$$$$
=4 f(t)^{4 N} 2 c(t)^{2}[1-c(t)]^{2}\left(4(1-\hat{f}(t))^{2} \hat{f}(t)^{6}\right.
$$$$
\left.+4(1-\hat{f}(t))^{3} \hat{f}(t)^{5}+\hat{f}(t)^{4}(1-\hat{f}(t))^{4}\right) \text {. }
$$

Similarly,

$\operatorname{Pr}\{X>t, L=0$, the root ring is segmented evenly $\}$

$=\operatorname{Pr}\{X>t \mid L=0$, the root ring is segmented evenly $\}$

$$
\times \operatorname{Pr}\{L=0\}
$$

$\times \operatorname{Pr}\{$ the root ring is segmented evenly | $L=0\}$

$$
\begin{aligned}
= & f(t)^{4 N} 2 c(t)^{2}[1-c(t)]^{2}\left(4(1-\hat{f}(t))^{2} \hat{f}(t)^{6}\right. \\
& \left.+4(1-\hat{f}(t))^{3} \hat{f}(t)^{5}+\hat{f}(t)^{4}(1-\hat{f}(t))^{4}\right) .(14)
\end{aligned}
$$

\section{Summing the above two equations gives}

$\operatorname{Pr}\{X>t, L=0$, the root ring is segmented $\}$

$$
\begin{aligned}
= & 10 f(t)^{4 N} c(t)^{2}[1-c(t)]^{2}\left(4(1-\hat{f}(t))^{2} \hat{f}(t)^{6}\right. \\
& \left.+4(1-\hat{f}(t))^{3} \hat{f}(t)^{5}+\hat{f}(t)^{4}(1-\hat{f}(t))^{4}\right) .(15)
\end{aligned}
$$

For $L=1$,

$\operatorname{Pr}\{X>t, L=1$, the root ring is segmented evenly $\}$

$$
\begin{array}{r}
=f(t)^{4 N} 4 c(t)^{3}[1-c(t)]\left(4(1-\hat{f}(t))^{2} \hat{f}(t)^{6}\right. \\
\left.+4(1-\hat{f}(t))^{3} \hat{f}(t)^{5}+\hat{f}(t)^{4}(1-\hat{f}(t))^{4}\right)
\end{array}
$$

and

$\operatorname{Pr}\{X>t, L=1$, the root ring is segmented unevenly $\}$

$$
=\left(\left[2 f(t)^{2}-f(t)^{4}\right]^{N} f(t)^{2 N}\right) 4 c(t)^{3}[1-c(t)] \times
$$

$\left[4(1-\hat{f}(t))^{2} \hat{f}(t)^{6}+4(1-\hat{f}(t))^{3} \hat{f}(t)^{5}+\hat{f}(t)^{4}(1-\hat{f}(t))^{4}\right]$.

Then summing the above two equations gives
$\operatorname{Pr}\{X>t, L=1$, the root ring is segmented $\}$

$$
\begin{array}{r}
=\left(\left[2 f(t)^{2}-f(t)^{4}\right]^{N} f(t)^{2 N}+f(t)^{4 N}\right) \\
\times 4 c(t)^{3}[1-c(t)]\left(4(1-\hat{f}(t))^{2} \hat{f}(t)^{6}\right. \\
\left.+4(1-\hat{f}(t))^{3} \hat{f}(t)^{5}+\hat{f}(t)^{4}(1-\hat{f}(t))^{4}\right) .(16)
\end{array}
$$

For $L=2$,

$\operatorname{Pr}\{X>t, L=2$, the root ring is segmented evenly $\}$

$$
\begin{array}{r}
=f(t)^{4 N} c(t)^{4}\left(4(1-\hat{f}(t))^{2} \hat{f}(t)^{6}\right. \\
\left.+4(1-\hat{f}(t))^{3} \hat{f}(t)^{5}+\hat{f}(t)^{4}(1-\hat{f}(t))^{4}\right)
\end{array}
$$

and

$P\{X>t, L=2$, the root ring is segmented unevenly $\}$

$$
\begin{array}{r}
=4\left[2 f(t)^{2}-f(t){ }^{4}\right]^{N} f(t)^{2 N_{c}} c(t)^{4}\left(4(1-\hat{f}(t))^{2} \hat{f}(t)^{6}\right. \\
\left.+4(1-\hat{f}(t))^{3} \hat{f}(t)^{5}+\hat{f}(t)^{4}(1-\hat{f}(t))^{4}\right) .
\end{array}
$$

Hence by summing the above two equations,

$\operatorname{Pr}\{X>t, L=2$, the root ring is segmented $\}$

$$
\begin{array}{r}
=\left(4\left[2 f(t)^{2}-f(t)^{4}\right]^{N} f(t)^{2 N}+f(t)^{4 N}\right) \\
\times c(t)^{4}\left(4(1-\hat{f}(t))^{2} \hat{f}(t)^{6}\right. \\
\left.+4(1-\hat{f}(t))^{3} \hat{f}(t)^{5}+\hat{f}(t)^{4}(1-\hat{f}(t))^{4}\right) \cdot(17)
\end{array}
$$

Then by summing Eqs. (15), (16) and (17):

$\operatorname{Pr}\{X>t$, the root ring is segmented $\}$

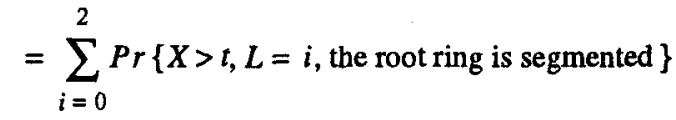

$$
=\left(4(1-\hat{f}(t))^{2} \hat{f}(t)^{6}+4(1-\hat{f}(t))^{3} \hat{f}(t)^{5}\right.
$$

$$
\begin{array}{r}
\left.+\hat{f}(t)^{4}(1-\hat{f}(t))^{4}\right) \times\left\{10 f(t)^{4 N} c(t)^{2}[1-c(t)]^{2}\right. \\
+\left(\left[2 f(t)^{2}-f(t)^{4}\right]^{N} f(t)^{2 N}+f(t)^{4 N}\right) 4 c(t)^{3}[1-c(t)] \\
\left.+\left(4\left[2 f(t)^{2}-f(t)^{4}\right]^{N} f(t)^{2 N}+f(t)^{4 N}\right) c(t)^{4}\right\} .(18)
\end{array}
$$

Case 2: $M \neq 2$. Note that the necessary condition for the segmented network to be reliable in this case is that: At most one CON is isolated. Then it can be shown that 
$\operatorname{Pr}\{X>t$, the root ring is segmented $\}$

$$
\begin{array}{r}
=\operatorname{Pr}\{X>t, \text { only one CON is isolated }\} \\
=\sum_{m=0}^{M}\left(\left[2 f(t)^{2}-f(t)^{4}\right]^{N}\right)^{M-m-1} f(t)^{2 N m} \\
\times\left(\begin{array}{l}
M \\
m
\end{array}\right) c(t)^{2(M-m)}[2 c(t)(1-c(t))]^{m} \\
\times\left(4(1-\hat{f}(t))^{2} \hat{f}(t)^{4 M-2}+4(1-\hat{f}(t))^{3} \hat{f}(t)^{4 M-3}\right. \\
\left.+(1-\hat{f}(t))^{4} \hat{f}(t)^{4 M-4}\right) \\
\times\left[f(t)^{2 N}(M-m)+m\left[2 f(t)^{2}-f(t)^{4}\right]^{N}\right] .(19)
\end{array}
$$

Then by combining Eqs. (11), (12), (18), and (19) results in the following theorem, which is an extension of Theorem 1.

\section{Theorem 3}

$$
\begin{aligned}
R(t)=\sum_{m=0}^{M}\left(\left[2 f(t)^{2}-f(t)^{4}\right]^{N}\right)^{M-m} f(t)^{2 m N} \\
\quad \times\left(\begin{array}{c}
M \\
m
\end{array}\right) c(t)^{2(M-m)}(2 c(t)[1-c(t)])^{m} \\
\quad \times\left((1-2 M) \hat{f}(t)^{4 M}+2 M \hat{f}(t)^{4 M-2}\right)+S(M, t),
\end{aligned}
$$

where $\quad S(M, t)=\operatorname{Pr}\{X>t$, the root ring is segmented $\}$, which is given by Eq. (18) if $M=2$ and by Eq. (19) if $M \neq 2$.

Note that Theorem 3 reduces to Theorem 1 when $M=1$. Theorem 2 in the previous section remains valid if $N$ DASs, which were used in that theorem, are replaced by $M N$ DASs in this section.

\section{Example 2}

Suppose that Example 1 in the previous section now is extended to include six CONs (i.e., $M=3$ ). Then the reliability values computed at $t=5,10$, and 50 days are given in Table 2.

Note that, by comparing Table 2 with Table 1 in the previous section, the dual homing network with $M=1$ is more reliable than that with $M=3$ even though their reliability upper bounds (Eqs. (9) and (13)) remain the same.
Table 2 - Reliability of Dual Ring and Extended Dual Homing Network

\begin{tabular}{|l|l|l|l|}
\hline$M=3$ & $t=5$ & $t=10$ & $t=50$ \\
\hline$M N=21$ & $0.9979^{1}$ & 0.9918 & 0.8261 \\
$(N=7)$ & $0.9947^{2}$ & 0.9795 & 0.6492 \\
\hline$M N=39$ & 0.9961 & 0.9848 & 0.7013 \\
$(N=13)$ & 0.9913 & 0.9669 & 0.5137 \\
\hline$M N=48$ & 0.9953 & 0.9814 & 0.6462 \\
$(N=16)$ & 0.9897 & 0.9609 & 0.4624 \\
\hline
\end{tabular}

1 computed from Eq. (13): upper bound for dual homing network

2 computed from Theorem 3: extended dual homing network

\section{Conclusion}

Closed form reliability functions for the FDDI dual homing networks are derived (Theorems 1 and 3). In most cases, dual homing is more reliable than dual ring; however, dual homing technique is beneficial only if the following conditions are met (Theorem 2):

- the number of DASs must be at least four

- the link reliability should be at least $\sqrt{2} / 2=.7071 \ldots$

- the root ring comprising CONs must be reliable

- the number of CON pairs on the root ring should be as small as possible

\section{References}

1. F. Ross, "FDDI - A Tutorial," IEEE Communications, 24(5): 10-17 (1986).

2. D. Logothetis and K. Trivedi, "Reliability Analysis of the FDDI Token Ring," IEEE Proceedings of the 16th Conference on LANs, Oct. 1991.

3. K. Ochettree, "Using Redundancy in FDDI Networks," IEEE Proceedings of the 15th Conference on LANs, Oct. 1990.

4. M. Willebeek-LeMair and A. Ruiz, "FDDI Network Availability Analysis," IEEE Proceedings of the 16th Conference on LANs, Oct. 1991. 